\title{
Surface composition and temperature of the TNO Orcus ${ }^{\star}$
}

\author{
M. A. Barucci ${ }^{1}$, F. Merlin ${ }^{1}$, A. Guilbert ${ }^{1}$, C. de Bergh ${ }^{1}$, A. Alvarez-Candal ${ }^{1}$, O. Hainaut ${ }^{2}$, A. Doressoundiram ${ }^{1}$, \\ C. Dumas ${ }^{2}$, T. Owen ${ }^{3}$, and A. Coradini ${ }^{4}$ \\ 1 LESIA, Observatoire de Paris, 92195 Meudon Principal Cedex, France \\ e-mail: antonella.barucci@obspm.fr \\ 2 ESO, Alonso de Cordova 3107, Vitacura, Casilla 19001, Santiago 19, Chile \\ 3 Institute or Astronomy, 2680 Woodlawn Drive, Honolulu, HI96822, USA \\ 4 INAF - IFSI, Via del Fosso del Cavaliere, 00133 Roma, Italy \\ Received 16 November 2007 / Accepted 19 December 2007
}

ABSTRACT

\begin{abstract}
Aims. The aim of this paper is to investigate the surface composition of the Transneptunian Object (TNO) Orcus. Methods. High quality observations have been carried out with the new instrument SINFONI at the Very Large Telescope (VLT) of ESO.

Results. Crystalline water ice, and possibly ammonia ice, have been found from spectroscopic observations of the TNO Orcus between 1.4 and $2.4 \mu \mathrm{m}$.

Conclusions. The existence of such ices on the surface of Orcus may indicate a renewal mechanism on the surface and geological activity. The presence of ammonia on the surface of Orcus, if confirmed, could have important implications for the composition of the primitive solar nebula and the formation of the TNO population.
\end{abstract}

Key words. Kuiper Belt - techniques: spectroscopic - methods: observational

\section{Introduction}

Transneptunian objects (TNOs) contain key information on how accretion proceeded in the early solar system. The study of physical properties of TNOs is fundamental to understand their origin and evolution. Only a few large TNOs have been discovered recently (Brown 2008). 90482 Orcus is a large TNO with a diameter estimated by Spitzer of $946 \pm 72.3 / 74.1 \mathrm{~km}$ and an albedo of $19.3 \%$ (Stansberry et al. 2008). Up to now only six TNOs or satellites of TNOs (Pluto, Charon, Eris, $2005 \mathrm{FY}_{9}$, Sedna and $2003 \mathrm{EL}_{61}$ ) have larger diameters than Orcus.

Orcus belongs to the Plutino dynamical class, as it is in the 3:2 resonance with Neptune with an orbit shaped similarly to that of Pluto. A satellite was discovered by HST observations in February 2007 (Brown \& Suer 2007) at 0.25 arcsec from Orcus with a magnitude difference of 2.7. Assuming an albedo similar to that of the primary, the magnitude suggests a quite substantial diameter of around $220 \mathrm{~km}$ for the satellite. Recent observations of the satellite orbit suggest a density for Orcus comparable to that of Charon (Brown 2007, private communication). Ortiz et al. (2006) determined a possible rotational period of $10.08 \pm 0.01$ $\mathrm{hr}$ for Orcus and an amplitude of $0.04 \pm 0.02$, considering only a single peak.

Spectroscopic observations in the near-infrared with the Galileo telescope (Fornasier et al. 2004) showed the presence of water ice at the surface of Orcus. Subsequent observations at ESO-VLT (de Bergh et al. 2005) suggested that the water ice was in the crystalline state. Both observations were modeled with mixtures of water ice and carbonaceous compounds.

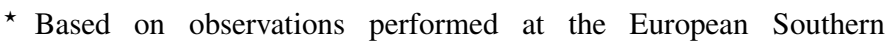
Observatory (ESO), proposal 178.C-0867.
}

Further $K$-band spectra taken with the Gemini telescope confirmed a water ice signature at $2 \mu \mathrm{m}$ corresponding to less than $50 \%$ of the surface fraction (Trujillo et al. 2005). The visible spectrum of Orcus is nearly flat (neutral color) and featureless (Fornasier et al. 2004; de Bergh et al. 2005).

In this paper we report one of the highest quality near infrared spectra obtained to date on the TNO population.

\section{Observations and data reductions}

Orcus was observed on January 24, 2007, in the near infrared with the $8 \mathrm{~m}$ Yepun telescope (ESO-VLT facility at the Paranal Observatory in Chile). We used the new instrument SINFONI (Spectrograph for INtegral Field Observations in the Near Infrared) to obtain simultaneous spectra in the $H$ and $K$ bands, in the no-AO mode. Orcus was observed for $100 \mathrm{~min}$, while its airmass ranged from 1.099 to 1.061 , and with a stable seeing of $0.5^{\prime \prime}$ throughout the exposure time. The solar analog, Landolt 98-978, was observed just before the object at airmass 1.1 with the same instrumental settings, in order to provide the needed calibration to correct the spectrum from the solar response and from the telluric features.

SINFONI slices the field of view into 32 image-slitlets which are re-directed toward the grating and then re-imaged on the detector. To improve our estimation of the background, the target was shifted on different positions of the SINFONI field between each $600 \mathrm{~s}$ exposure. The reduction was performed using the SINFONI pipeline version 1.6 which provides a robust wavelength calibration (accuracy of $0.0005 \mu \mathrm{m}$ per pixel) and a good correction of the skylines.

The processed spectra were extracted using QFitsView, a $3 \mathrm{D}$-visualization tool developed for SINFONI. The extraction is 


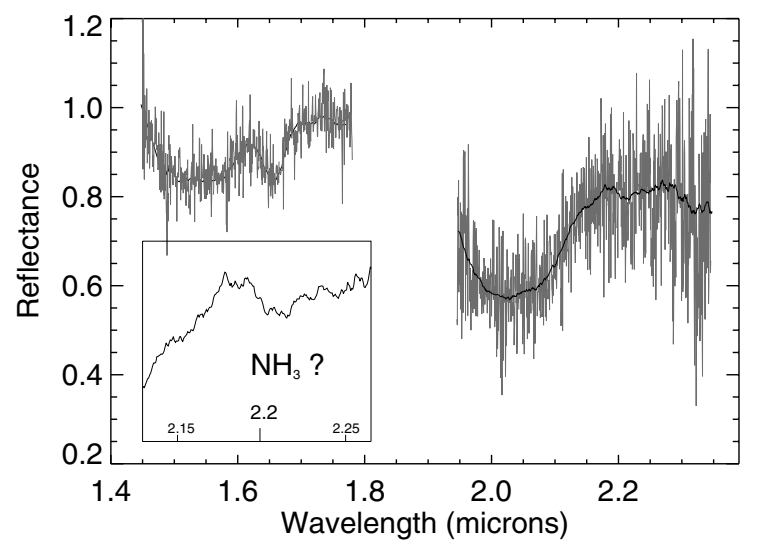

Fig. 1. Reflectance spectrum of Orcus $(R=1500)$, normalized to unity at $1.55 \mu \mathrm{m}$, with the smoothed spectrum $(R=100)$. The strong absorption bands are due to crystalline water ice. A weak band is present around $2.2 \mu \mathrm{m}$, reported at larger scale in the small square, and tentatively assigned to hydrated ammonia (see text). Other bands are noted beyond $2.25 \mu \mathrm{m}$, but they are in lower signal to noise level portions of the spectrum.

performed using an aperture selected as a compromise between the maximum gathered flux and the minimum residual sky contribution, and we use in this case an aperture corresponding to a 1 " radius.

The target's spectrum was then divided by the solar analog's spectrum. The resulting spectrum (Fig. 1) was finally degraded to a resolution of about 100 by means of a running box to increase the $\mathrm{S} / \mathrm{N}$ ratio.

\section{Analysis and spectral models}

The spectrum of Orcus, of high $\mathrm{S} / \mathrm{N}$ and unique quality, shows very deep $\mathrm{H}_{2} \mathrm{O}$ absorptions at 1.5 and $2.0 \mu \mathrm{m}$ (Fig. 1). The clear feature at $1.65 \mu \mathrm{m}$ indicates unambiguously the presence of the crystalline phase of water ice. The $1.65 \mu \mathrm{m}$ band is large and deep (12\%), as on Charon (Cook et al. 2007), Quaoar (Jewitt \& Luu 2004), $2003 \mathrm{EL}_{61}$ (Merlin et al. 2007), and icy satellites of giant planets (i.e., Grundy et al. 2006; Emery et al. 2005).

Grundy \& Schmitt (1998) showed that the wavelength of the band of crystalline water ice at $1.65 \mu \mathrm{m}$ strongly depends on temperature. This band was used to determine the temperature of crystalline water ice on other TNOs whose spectrum is dominated by water ice (on Charon, by Cook et al. 2007; or on the TNO 136108 (2003 EL 61 ), by Merlin et al. 2007). We use the method described in Merlin et al. (2007) to determine the central position of the band in Orcus spectrum. We find the minimum of the band close to $1.6561 \pm 0.0009 \mu \mathrm{m}$, which corresponds to a temperature of $44 \mathrm{~K}$. However, taking into account a possible contribution of amorphous water ice (see further) and possible effects due to irradiation (see Merlin et al. 2007, for more details about temperature constraints) this value of $44 \mathrm{~K}$ should be considered as an upper limit to the temperature.

In addition, an absorption feature appears in the spectrum of Orcus around $2.2 \mu \mathrm{m}$ (see Fig. 1). This band has a depth of $10 \%$ and is centered around 2.216 $\pm 0.001 \mu \mathrm{m}$. This feature is somewhat similar to that observed on Charon by Brown \& Calvin (2000), Dumas et al. (2001), and Cook et al. (2007), on Miranda by Bauer et al. (2002) and suggested on Quaoar by Jewitt \& Luu (2004), and attributed to a combination of pure ammonia and ammonia hydrate (Brown \& Calvin 2000). It should be noted that, for Charon, the ammonia is not distributed uniformly; the

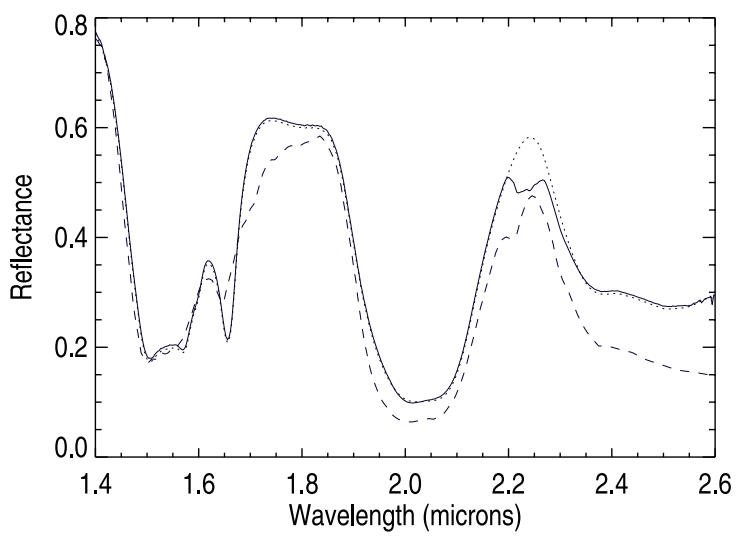

Fig. 2. Reflectance spectra of some of the ices used in our models. Dilution of ammonia in crystalline water ice induces shifts in the wavelength of the ammonia band near 2.21 microns. Increasing the amount of diluted ammonia in crystalline water ice tends to reduce the depth of the band at 1.65 micron. Dotted line represents $\mathrm{H}_{2} \mathrm{O}$ ice in crystalline phase, continuous line is an intimate mixture of pure crystalline $\mathrm{H}_{2} \mathrm{O}$ ice and $3 \%$ of pure $\mathrm{NH}_{3}$ and dashed line is $\mathrm{NH}_{3}(1 \%)$ diluted in crystalline $\mathrm{H}_{2} \mathrm{O}$ ice.

band is more noticeable on the leading hemisphere and there is a slight difference in the wavelength positions (2.1995 and $2.2131 \mu \mathrm{m}$, respectively) between the two hemispheres (Cook et al. 2007). Concerning Quaoar, the presence of an absorption feature at $2.2 \mu \mathrm{m}$ has been confirmed by Schaller \& Brown (2007), but is assigned instead to methane ice. It should also be noted that ammonia has not been detected on any icy satellite of the outer planets other than Miranda.

$\mathrm{NH}_{3}$ ice has two bands in the near-infrared range, the first at $2.0 \mu \mathrm{m}$, completely hidden by the water ice absorption band in our case, and a second one between 2.24 and $2.21 \mu \mathrm{m}$, depending on the hydration state of the ammonia (see Fig. 3 of Schmitt et al. 1998; and Moore et al. 2007). The absorption band of pure $\mathrm{NH}_{3}$ ice appears at $2.24 \mu \mathrm{m}$ while the center of the band we observe is close to $2.21 \mu \mathrm{m}$, which would imply that $\mathrm{NH}_{3}$, if present on the surface of Orcus, would be hydrated. As methane ice also has a band around $2.2 \mu \mathrm{m}$, we considered the possibility that methane is present on Orcus as well.

To investigate the surface properties of this object, we ran a radiative transfer model, based on the Hapke theory (1981 and 1993). To account for the presence of the absorption feature near $2.2 \mu \mathrm{m}$, we first considered ammonia in pure or diluted state with water ice (see Fig. 2). We find that only small amounts of ammonia ice diluted in crystalline water ice must be present to conserve the $1.656 \mu \mathrm{m}$ band. The optical constants of ammonia come from Roush (personal communication) for the diluted states and from Schmitt et al. (1998) for the pure state.

A mixture of crystalline and amorphous water ice, combined with ammonia ices, provides the best fit of the absorption bands. The optical constants of water ice used in our models are those of amorphous water ice at $38 \mathrm{~K}$ from Schmitt et al. (1998) and crystalline water ice at $40 \mathrm{~K}$ from Grundy \& Schmitt (1998). With only these compounds, however, we cannot reproduce the blue slope of the spectrum in the near infrared. Therefore, as was done for Charon by Cook et al. (2007), we introduce an additional "blue component" in our model. Several phyllosilicates show this kind of behavior (Trujillo et al. 2007, including a weak feature at $2.2 \mu \mathrm{m}$, but they also present some other absorption bands. For the sake of simplicity, we choose to introduce in our 


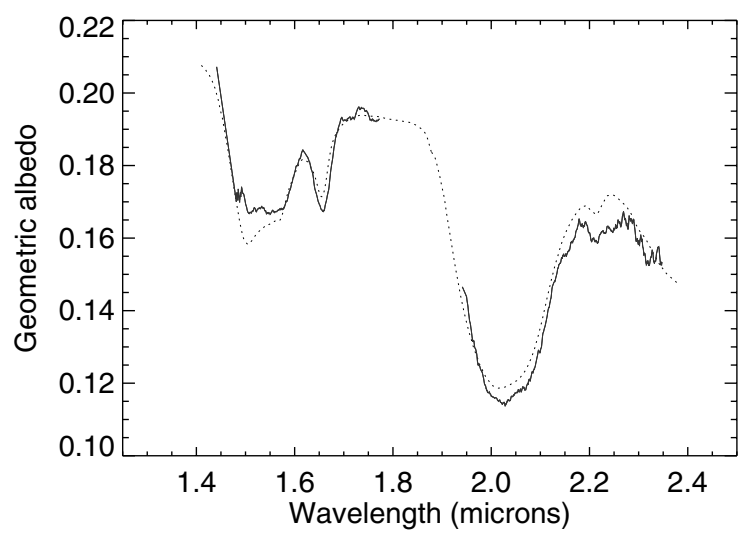

Fig. 3. The dotted line superimposed on the observed spectrum (solid line) is the synthetic spectrum which gives the best $\chi^{2}$. It corresponds to a spatial mixture of crystalline water ice, amorphous water ice, ammonia hydrate and a "blue" component (see text). The geometric albedo of Orcus is from Stansberry et al. (2008).

models a component that is both "blue" (linear slope without any absorption) and dark (to account for the low albedo of Orcus).

We use a mean square algorithm to obtain the model that gives the best agreement with the observations. The best fit (reported in Fig. 3) is obtained when including water ice in both states (crystalline and amorphous, respectively $15 \%$ and $17 \%$ with grain sizes of 50 and 20 microns), ammonia diluted (1\%) in crystalline water ice (5\% with large grain size of $250 \mu \mathrm{m})$, and large amounts of the blue component (63\% with grain size of $40 \mu \mathrm{m})$. The blue component that provides the best fit is relatively dark and has a blue slope of about 5\%/100 nm. The fit of the ammonia band absorption is not perfect but the general behavior of the band near $2.2 \mu \mathrm{m}$ is reproduced, as well as the absorption band of crystalline water ice at $1.65 \mu \mathrm{m}$, and this is the best model that can be obtained using the available optical constants for ammonia hydrates. It should be noted that only two sets of optical constants exist for ammonia hydrates (both from Roush) and that they were both derived from reflectance spectra of not very well characterized samples of $\mathrm{NH}_{3}: \mathrm{H}_{2} \mathrm{O}$ at $80 \mathrm{~K}$ (from Brown et al. 1988).

We also ran a model in which ammonia is replaced by methane ice (see Fig. 4). The optical constants for methane are from Quirico \& Schmitt (1997) and correspond to a temperature of $40 \mathrm{~K}$. As shown in Fig. 4, the band of methane around $2.2 \mu \mathrm{m}$ is too narrow and not centered at the right wavelength, and the fit beyond 2.25 micron is not as good as with the ammonia. Moreover the band due to methane at $1.724 \mu \mathrm{m}$ is not present in the observed spectrum of Orcus. We cannot exclude however the presence of small amounts of methane on the surface of Orcus.

\section{Discussion}

The analogies between Charon and Orcus noted in de Bergh et al. 2005 (orbital properties, neutral color in the visible, deep water ice absorptions) are now strengthened by the unambiguous detection of crystalline water ice on Orcus, the detection of a feature at $2.2 \mu \mathrm{m}$ somewhat similar to that observed on Charon, and the recent measurements of Orcus' density and visible albedo (about 0.2 compared to $0.36-0.39$ for Charon) by Stansberry et al. (2008). Only small amounts of methane could be present

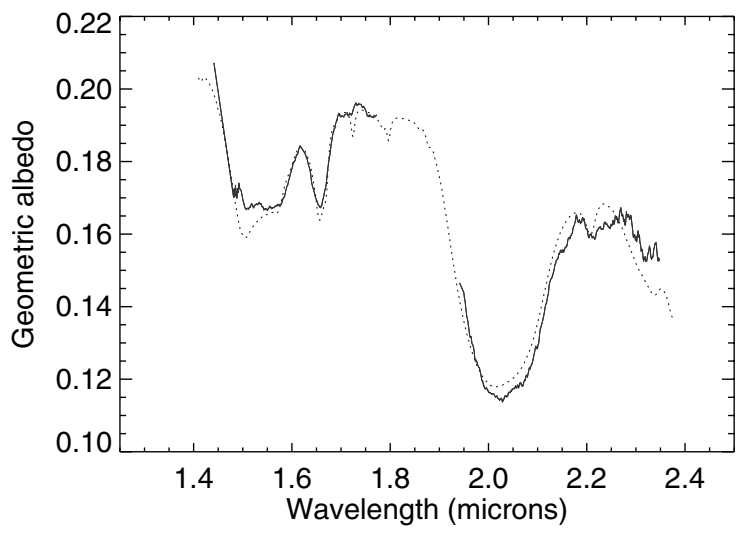

Fig. 4. Model including methane ice instead of ammonia. The dotted line superimposed on the spectrum (solid line) is the synthetic spectrum which gives the best $\chi^{2}$ (including $3.3 \% \mathrm{CH}_{4}$ with grain size of $450 \mu \mathrm{m}$ ). The geometric albedo of Orcus is from Stansberry et al. (2008).

on the surface of Charon (Cook et al. 2007), and this seems to also be the case for Orcus.

Our models indicate that, as for Charon, the best candidate to explain the $2.2 \mu \mathrm{m}$ feature is some ammonia hydrate. However, this identification is not unique as it relies only on one feature (which is also the case for Charon); furthermore, this feature is quite weak and needs confirmation. Still higher $\mathrm{S} / \mathrm{N}$ observations are obviously required.

As already discussed by Jewitt \& Luu (2004) and Cook et al. (2007), the presence of crystalline water ice and ammonia hydrates on the surface of a TNO would tend to argue for an efficient surface renewal mechanism. Indeed, extrapolation of laboratory experiments on water ice irradiation to the conditions of a TNO at $40 \mathrm{AU}$ indicates that crystalline water ice on the surface of TNOs should be completely amorphized by irradiation in about $10^{7}$ years (Cooper et al. 2003). In recent works (Barucci et al. 2008; Barkume et al. 2008), TNOs with spectra showing water-ice absorptions measured with high $\mathrm{S} / \mathrm{N}$ have been shown to have the $1.65 \mu \mathrm{m}$ band due to the presence of $\mathrm{H}_{2} \mathrm{O}$ ice in the crystalline phase. While crystalline water ice is neither expected at these lower temperatures nor should it be stable against cosmic-ray and UV bombardment, crystalline water ice appears to be ubiquitous in the outer solar system. Brown et al. (2007) suggested that the deep water-ice absorption on the TNO spectra has to be a function of a relatively short surface exposure age implying a relatively recent collision for these objects. Both internal and external surface renewal mechanisms have been considered to explain the presence of crystalline water ice on TNOs: heating accomplished by micrometeoritic or larger impacts (impact gardening) or cryovolcanism, even if it is clear that our current understanding of the physics of the crystalline/amorphous phase transition is not complete.

Concerning ammonia, Strazzulla \& Palumbo (1998) demonstrated that ammonia hydrates are easily destroyed by energetic radiation, and Cooper et al. (2003) showed that at $40 \mathrm{AU}$ from the Sun it would happen in less than $10^{7}$ years. An external source of ammonia was therefore hypothesized for Charon, as its neighbour Pluto is very rich in nitrogen (e.g., Dumas et al. 2001), whereas cryovolcanism was considered for Quaoar (Jewitt \& Luu 2004) and for Charon (Cook et al. 2007). Recent models of the interiors of TNOs show that cryovolcanism, which is considered the most probable mechanism to explain geological activity 
on some satellites of the outer planets (and which would be facilitated by the presence of ammonia in the interior of these bodies), may indeed be possible for TNOs larger than about $1000 \mathrm{~km}$ (McKinnon et al. 2008).

A firm detection of ammonia on the surface of an isolated TNO such as Orcus would also have important implications on the composition of the primitive solar nebula in regions of low density (far from the Sun), allowing us to determine if nitrogen was present mainly in the form of $\mathrm{N}_{2}$ or $\mathrm{NH}_{3}$ at more than 40 AU from the Sun. Early models of solar nebula condensation in the outer solar system indicated that $\mathrm{NH}_{3}$ may have been an important condensate (Lewis 1972), while more recent models predict that, due to possible kinetic inhibition of $\mathrm{N}_{2}$ reduction in the solar nebula, most of the nitrogen was present as $\mathrm{N}_{2}$ (Lewis \& Prinn 1980). Ammonia may be present on Miranda, as already mentioned (Bauer et al. 2002). However, the case of icy satellites of the outer solar system is peculiar, since they formed in the warm and dense subnebulae of each proto-gasgiant planet. Ammonia has been detected in a few bright comets (Bockelée-Morvan et al. 2004). Its photodissociation products, $\mathrm{NH}$ and $\mathrm{NH}_{2}$, have been detected in many more comets, including Jupiter family comets. The abundance of ammonia in the nucleus is of the order of $0.5 \%$ relative to water while the amount of $\mathrm{N}_{2}$ in comets, if present at all, is much lower (see review of Bockelée-Morvan et al. 2004). It is therefore very important to search for nitrogen compounds on the surface of TNOs and see which ones dominate, keeping in mind that the surface composition of the object does not necessarily reflect its bulk composition (in particular, as ammonia is easily destroyed, much larger amounts of ammonia could be present inside an object on which small amounts of ammonia are detected). The detection of ammonia is also very important for the geological study of TNOs, particularly for the low-melting point of cryovolcanism that it would imply.

To secure our detection of ammonia on Orcus requires still higher $\mathrm{S} / \mathrm{N}$ spectra. This may be possible in the near future at the VLT by using the same instrument (SINFONI) with the adaptive optics system and the laser guide star, which are both now available. More laboratory work on ammonia hydrates, and in particular measurements of optical constants, is also required.

Acknowledgements. We are very grateful to T. Roush to have provided the optical constants for ammonia hydrates and to J. Emery for his helpful review and suggestions.

\section{References}

Barkume, K. M., Brown, M. E., \& Shaller, E. L. 2008, AJ, in press

Barucci, M. A., Brown, M. E., Emery, J. P., \& Merlin, F. 2008, in The Solar System Beyond Neptune, ed. M. A. Barucci, H. Boehnhardt, D. P. Cruikshank, \& A. Morbidelli (Tucson: Univ. of Arizona Press), in press

Bauer, J. M., Roush, T. L., Geballe, T. R., et al. 2002, Icarus, 158, 178

Brown, M. E. 2008, in The Solar System Beyond Neptune, ed. M. A. Barucci, H. Boehnhardt, D. P. Cruikshank, \& A. Morbidelli (Tucson: Univ. of Arizona Press), in press

Brown, M. E., \& Calvin, W. M. 2000, Science, 287, 107

Brown, M. E., \& Suer, T. A. 2007, IAUC, 8812

Brown, M. E., Barkume, K. M., Ragozzine, D., \& Shaller, E. L. 2007. Nature, 446, 294

Brown, R. H., Cruikshank, D. P., Tokunaga, A. T., et al. 1988, Icarus, 74, 262 de Bergh, C., Delsanti, A., Tozzi, G. P., et al. 2005, A\&A, 437, 1115

Bockelée-Morvan, D., Crovisier, J., Mumma, M. J., \& Weaver, H. A. 2004, in Comets II, ed. M. C. Festou, H. U. Keller, \& H. A. Weaver (Tucson: Univ. of Arizona Press), 391

Cook, J. C., Desch, S. J., Roush, T. E., et al. 2007, ApJ, 663, 1406

Cooper, J. F., Christian, E. R., Richardson, J. D., \& Wang, C. 2003, Earth, Moon \& Planets, 92, 261

Dumas, C., Terrile, R. J., Brown, R. H., et al. 2001, AJ, 121, 1163

Emery, J. P., Burr, D. M., Cruikshank, D. P., et al. 2005, A\&A, 435, 353

Fornasier, S., Dotto, E., Barucci, M. A., \& Barbieri, C. 2004, A\&A, 422, L43

Hapke, B. 1981, J. Geophy. Res., 86, 3039

Hapke, B. 1993, Theory of reflectance and emittance spectroscopy, in Topics in remote sensing 3 (Cambridge University Press)

Grundy, W. M., \& Schmitt, B. 1998. J. Geophys. Res., 103, 25809

Grundy, W. M., Young, L. A., Spencer, J. R., et al. 2006, Icarus, 184, 543

Jewitt, D. C., \& Luu, J. 2004, Nature, 432, 731

Lewis, J. S. 1972, Icarus, 16, 241

Lewis, J. S., \& Prinn, R. G. 1980, ApJ, 238, 357

McKinnon, W. B., Prialnik, D., Stern, A., \& Coradini, A. 2008, in The Solar System Beyond Neptune, ed. M. A. Barucci, H. Boehnhardt, D. P. Cruikshank, \& A. Morbidelli (Tucson: Univ of Arizona Press), in press

Merlin, F., Guilbert, A., Dumas, C., et al. 2007, A\&A, 466, 1185

Moore, M. H., Ferrante, R. F., Hudson, R. L., \& Stone, J. N. 2007, Icarus, 190, 260

Ortiz, J., Guitierrez, P., Santos-Sanz, P., et al. 2006, A\&A, 447, 1131

Quirico, E., \& Schmitt, B. 1997, Icarus, 127, 354

Schaller, E. L., \& Brown, M. E. 2007, ApJ, 670, L49

Schmitt, B., Quirico, E., Trotta, F., \& Grundy, W. M. 1998, in Solar System Ices, ed. B. Schmitt, C. de Bergh, \& M. Festou (Kluver Acad. Publisher), 199

Stansberry, J., Grundy, W., \& Brown, M. 2008, in The Solar System Beyond Neptune, ed. M. A. Barucci, H. Boehnhardt, D. P. Cruikshank, \& A. Morbidelli (Tucson: Univ of Arizona Press), in press

Strazzulla, G., \& Palumbo, M. E. 1998, Planet. \& Space Sci., 46, 1339

Trujillo, C. A., Brown, M. E., Rabinowitz, D. L., \& Geballe, T. R. 2005, ApJ, 627, 1057

Trujillo, C. A., Brown, M. E., \& Barkume, K. M. 2007, ApJ, 655, 1172 